\title{
Preventive care for a 35-year-old woman with inflammatory bowel disease
}

\author{
Neeraj Narula MD, A. Hillary Steinhart MD MSc
}

\author{
Cite as: CMAJ 2017 February 13;189:E243-4. doi: 10.1503/cmaj.160640
}

\begin{abstract}
A 35-year-old woman with ulcerative colitis presents to her primary care physician and asks if she should receive the influenza vaccine. After left-sided ulcerative colitis was diagnosed 17 years ago and did not respond to mesalamine treatment, the patient achieved clinical remission with prednisone and has been taking azathioprine for 16 years. She has not seen a gastroenterologist since moving three years ago.
\end{abstract}

\section{Is it safe for this patient to receive the influenza vaccine? What about other vaccines?}

Patients with inflammatory bowel disease (IBD) may not undergo adequate vaccination for a variety of reasons, including concern for adverse events, lack of awareness that vaccination was indicated, and fear that vaccination may trigger a relapse of disease. ${ }^{1}$ For patients using immunosuppressive therapies, such as corticosteroids, immunomodulators and biologic agents, live vaccines are contraindicated and should not be given until three months after such therapies are stopped. ${ }^{2}$ Live vaccines available for use in Canada include measles-mumps-rubella, live attenuated influenza vaccine, varicella, herpes zoster, rotavirus, smallpox, Bacillus Calmette-Guérin, yellow fever and orally administered typhoid vaccine. ${ }^{2}$ Thus, it is best for patients with IBD to undergo vaccination before starting immunosuppressive therapies.

For patients using immunosuppressive therapies, it is safe to administer inactive vaccines, consistent with the Canadian Immunization Guide, although some patients may not mount a sufficient protective response. ${ }^{3}$ In addition to the annual inactivated influenza vaccine, these patients should be offered pneumococcal vaccine (including a one-time revaccination after five years in patients older than 65 years of age or for patients using immunosuppressive therapies), tetanus and diphtheria vaccine every 10 years, and the human papillomavirus (HPV) vaccine for female patients between the ages of 11 and 26 years. ${ }^{3}$ Hepatitis B vaccine should be offered if a patient is not immune. Other vaccines should be offered as appropriate, such as meningococcal vaccine for students. ${ }^{2,3}$

\section{What other preventive measures should be considered for this patient?}

Patients with IBD have been found to have an increased risk of cervical dysplasia and cervical cancer, particularly if they are using immunomodulators. ${ }^{3,4} \mathrm{~A}$ population-based study reported elevated incident rate ratios (IRRs) in patients with Crohn disease: 1.28 (95\% confidence interval $[\mathrm{Cl}] 1.13-1.45)$ for high-grade squamous intraepithelial lesions and $1.53(95 \% \mathrm{Cl}$ 1.04-2.27) for cervical cancer, compared with women in the general population. ${ }^{4}$ In ulcerative colitis, the IRR for high-grade squamous intraepithelial lesions was also elevated, $\left(1.12\right.$ [95\% Cl 1.01-1.25]), but not for cervical cancer. ${ }^{4}$ This increase was largely due to patients with a history of thiopurine or tumour necrosis factor (TNF) $\alpha$ antagonist use. For this reason, women with IBD who use immunosuppressive therapies should undergo annual screening for cervical cancer with a Papanicolaou (Pap) test, instead of every three years as recommended for Canadians at average risk for cervical cancer., 5 Human papilloma virus vaccination should also be offered when age appropriate. ${ }^{5}$

An increased risk of skin cancer has been seen in patients with IBD. Patients who used thiopurines had an increased risk (odds ratio [OR] 1.85 [95\% Cl 1.66-2.05) for nonmelanoma skin cancer, such as squamous cell and basal cell carcinoma, compared with patients who had never used these therapies. ${ }^{7}$ Patients who use TNF $\alpha$ antagonists, such as infliximab or adalimumab, had an increased risk (OR 1.88 [95\% Cl 1.08-3.29]) for melanoma skin cancer compared with patients who were not exposed to these agents. ${ }^{7}$ Thus, patients with IBD who use immunosuppressive therapies should undergo monitoring for skin cancer. Although there is no consensus on the monitoring interval, some experts suggest that patients using these therapies undergo annual dermatologic screening. ${ }^{8}$ Guidance should be provided to all patients on measures to reduce the risk of skin cancer, such as avoiding prolonged sun exposure and using skin protection, such as sunscreen and sun-protective clothing. ${ }^{8}$

Patients with Crohn disease who smoke are at increased risk of complications including internal penetrating disease and need for surgery. ${ }^{9}$ Smoking cessation has been shown to decrease clinical relapses and the need for corticosteroids. ${ }^{3}$ Active smokers with Crohn disease should be counselled about the effect of smoking on their disease, and all smokers (with or without Crohn disease) should be offered support for smoking cessation should they show an interest.

\section{When does the patient require colonoscopy for surveillance?}

Patients with ulcerative colitis and colonic Crohn disease have an elevated risk of colon cancer. Guidelines recommend that these patients begin a screening colonoscopy program eight years after diagnosis, although patients with ulcerative colitis that does not 
extend beyond the left side of the colon may wait 15 years from the time of diagnosis. ${ }^{10}$ Patients with ulcerative proctitis or isolated ileal Crohn disease do not have an increased risk of colon cancer and can undergo screening as average-risk patients. ${ }^{11}$ Screening colonoscopy should include multiple biopsies throughout the colon to examine for dysplastic changes or be performed with dye, such as methylene blue, to highlight potential areas of dysplasia. ${ }^{12}$ In addition, these patients should undergo surveillance at an interval of every one to three years after the initial screening. ${ }^{10}$ Certain conditions may merit more frequent surveillance. For example, patients who have concurrent primary sclerosing cholangitis should have a colonoscopy at the time of diagnosis and yearly thereafter owing to their even greater risk of colon cancer. Patients with a history of lowgrade dysplasia who opt against colectomy also merit more frequent surveillance. ${ }^{12}$

Box 1 summarizes preventive measures for patients with IBD.

Box 1: Summary of preventive measures for patients with inflammatory bowel disease

- Vaccination status: Ensure vaccinations are up to date, including inactivated influenza (annual), pneumococcal (one-time revaccination after 5 years in patients older than 65 years of age or for patients using immunosuppressive therapies), tetanus and diphtheria (every 10 years), and human papillomavirus (when age appropriate) ${ }^{3}$

- Annual Papanicolaou testing for female patients ${ }^{5}$

- Annual skin cancer surveillance for patients taking immunosuppressive agents and guidance on measures for sun protection $^{8}$

- Smoking cessation counselling

- Referral for colonoscopy surveillance if disease duration is 8 years or longer (ulcerative or Crohn colitis), or 15 years or longer if ulcerative colitis is limited to the left side of the colon ${ }^{10}$

\section{The case revisited}

The patient was offered inactivated influenza vaccine because she was using azathioprine. She was also offered pneumococcal vaccine and arranged to have a Pap test at her next visit. Because it had been more than 17 years since the time of her diagnosis, the patient was referred to a gastroenterologist for colonoscopy. Her colonoscopy showed evidence of quiescent ulcerative colitis on multiple biopsies, without dysplasia, and she continued azathioprine for maintenence therapy.

\section{References}

1. Narula N, Dhillon AS, Chauhan U, et al. An audit of influenza vaccination status in adults with inflammatory bowel disease. Can J Gastroenterol 2012;26:593-6.

2. National Center for Immunization and Respiratory Diseases. General recommendations on immunization - recommendations of the advisory committee on immunization practices (ACIP). MMWR Recomm Rep 2011;60:1-64.

3. Sinclair JA, Wasan SK, Farraye FA, et al. Health maintenance in the inflammatory bowel disease patient. Gastroenterol Clin North Am 2012;41:325-37.

4. Rungoe C, Simonsen J, Riis L, et al. Inflammatory bowel disease and cervical neoplasia: a population-based nationwide cohort study. Clin Gastroenterol Hepatol 2015;13:693-700e.1.

5. Nguyen ML, Flowers L. Cervical cancer screening in immunocompromised women. Obstet Gynecol Clin North Am 2013;40:339-57.

6. Canadian Task Force on Preventive Health Care. Recommendations on screening for cervical cancer. CMAJ 2013;185:35-45.

7. Long MD, Martin CF, Pipkin CA, et al. Risk of melanoma and nonmelanoma skin cancer among patients with inflammatory bowel disease. Gastroenterology 2012;143:390-9.e1.

8. Long MD, Kappelman MD, Pipkin CA. Nonmelanoma skin cancer in inflammatory bowel disease: a review. Inflamm Bowel Dis 2011;17:1423-7.

9. Louis E, Michel V, Hugot JP, et al. Early development of stricturing or penetrating pattern in Crohn's disease is influenced by disease location, number of flares, and smoking but not by NOD2/CARD15 genotype. Gut 2003;52:552-7.

10. Leddin D, Hunt R, Champion M, et al. Canadian Association of Gastroenterology and the Canadian Digestive Health Foundation: Guidelines on colon cancer screening. Can J Gastroenterol 2004;18:93-9.

11. Ekbom A, Helmick C, Zack M, et al. Increased risk of large-bowel cancer in Crohn's disease with colonic involvement. Lancet 1990;336:357-9.

12. Laine L, Kaltenbach T, Barkun A, et al. SCENIC international consensus statement on surveillance and management of dysplasia in inflammatory bowel disease. Gastroenterology 2015;148:639-51.e28.
Competing interests: A. Hillary Steinhart reports grants and personal fees from Abbvie; personal fees from Janssen, Takeda, Pfizer, Merck, Pharmascience, Shire, Allergan and Ferring; and grants from Genentech, Roche, Red Hill Biopharma, Centocor, Arena Pharmaceuticals and Celgene outside the submitted work. No other competing interests were declared.

This article has been peer reviewed.

The clinical scenario is fictional.
Affiliations: Department of Medicine (Division of Gastroenterology) and Farncombe Family Digestive Health Research Institute (Narula); McMaster University, Hamilton, Ont.; Zane Cohen Centre for Digestive Diseases (Narula), Mount Sinai Hospital IBD Group, Toronto, Ont., Canada Division of Gastroenterology, Department of Medicine (Narula), University of Toronto, Toronto, Ont.; Department of Gastroenterology (Steinhart), Mount Sinai Hospital, Toronto, Ont.
Contributors: Neeraj Narula conceived and designed the article. Hillary Steinhart drafted the article. Both of the authors revised the article for important intellectual content and approved the version to be published.

Correspondence to: Neeraj Narula, neeraj.narula@medportal.ca 